\title{
Factors Influencing the Parenting Stress of Mothers
}

\author{
Kim Sunmi \\ Lecturer, College of Nursing, Chungnam National University, Daejeon, \\ Republic of Korea \\ Sunmik204@gmail.com
}

\begin{abstract}
This study was conducted to identify the effects of parenting effectiveness, husband's parenting cooperation, and social support on a mother's parenting stress. The data were collected from August 2 to 30, 2018 by using a structured questionnaire. There was a total of 279 subjects from the online community and the data were analyzed using the SPSS 21 program. The objectives of the study were the following: determine if there is a difference in parenting stress according to the characteristics of the research subject; identify the relationships between key variables; identify the factors that influence parenting stress. According to the results of the study, the mother's parenting stress was influenced by the distress of her parents, the adverse functional interaction of her parents and children, and the effectiveness of parenting in difficult children. These results are meaningful in that they may be used as basic data for various studies that aim to ease the parenting stress of mothers raising infants.
\end{abstract}

Keywords: Husband's parenting cooperation, Parenting effectiveness, Social support, Parenting stress

\section{Introduction}

As the birth rate in Korea continues to decline, interest in childbirth is increasing [1]. Parenting stress can be suggested as a factor that adversely affects the fertility rate, and parenting stress is a key mechanism that influences parenting behavior [2]. It is also a factor that should be taken into consideration as it can cause mental health problems such as depression [3]. Married women experience difficulties in life while working and fulfilling their duties as a parent at the same time, so it may be an important matter to understand parenting stress [4]. Parenting stress is a cognitive factor in the actual difficulty and burden of parents while raising their children [5]. In addition, there are various factors affecting parenting stress so it is meaningful to approach it systematically to identify the influencing factors. In this study, specific parenting stress factors were identified by dividing the variables into microsystem, mesosystem, and exosystem from the ecological perspective of Mattaini and Lowery [6]. The microsystem identified a mother's parenting effectiveness, the mesosystem identified the husband's parenting cooperation, and finally, the exosystem identified social support. In this study, parenting stress was subdivided into parent pain, parent-child interaction, difficult children, and ecological theory [6], and the effects of these factors on parenting stress may provide data for parenting intervention studies and policy studies.

Article history:

Received (April 5, 2021), Review Result (June 10, 2021), Accepted (August 15, 2021) 
(1) Identify differences in parenting stress according to the general characteristics of the subject.

(2) Identify the relationship between parenting effectiveness, husband's parenting cooperation, and support.

(3) Identify the influencing factors of parenting stress.

\section{Methods}

\subsection{Subject and protocol}

This study was conducted to analyze the factors affecting the mother's parenting stress. As for the research model, a validated scale was put in the center of the ecological perspective. Data were collected online from the parenting-related communities in four regions including special cities, metropolitan cities, and city units. The data were collected from August 2 to 31, 2018 and the final analysis included a total of 279 subjects.

\subsection{Ethical considerations}

This study was conducted by the Institutional Review Board (MC18QCSE0061) of C University. The maintenance of personal confidentiality and guarantee of anonymity were explained to the participants and the collected data were secured by using a lock and password.

\subsection{Measurement tools}

\subsubsection{Parenting effectiveness}

K-EGSCP was used in this study. This tool was made by Meunier and Roskam's Echelle Globale du Sentiment de Compétence Parentale (EGSCP) and translated, modified, and used by Sung and Baek (2011) [7] to verify the adequacy of the factor structure theory through exploratory and verifiable factor analysis with Korean parents.

\subsubsection{Husband's parenting cooperation}

The husband's parenting cooperation in this study used a translation by Choi et al. (2018) [8] of the Parent Alliance Inventory (PAI), developed by Abidin (1988).

\subsubsection{Support}

The social support of this study was used by Kim (2012) [9] and the tool developed by Park (1985) was modified.

\subsubsection{Parenting stress}

The parenting stress of this study was based on PSI (Parent Stress Index) developed by Abidin (1995) and used a scale summarized by Jeong et al. (2020) [10] in Korean style.

\subsubsection{General characteristic}

The demographic characteristics included age, educational attainment, marriage period, number of children, environmental satisfaction, availability of childcare facilities, employment type, and income satisfaction. A total of nine questions were asked [Table 1]. 
Table 1. Measuring tools and reliability

\begin{tabular}{|c|c|c|c|}
\hline & \multirow{2}{*}{ Variable } & \multicolumn{2}{|c|}{ Cronbach's $\alpha$} \\
\hline & & Total & subscales \\
\hline \multirow{5}{*}{$\begin{array}{l}\text { Parenting } \\
\text { effectiveness }\end{array}$} & Affection & \multirow{5}{*}{.94} & .82 \\
\hline & Daily system organization & & .84 \\
\hline & Discipline & & .82 \\
\hline & Game & & .86 \\
\hline & Education & & .88 \\
\hline \multicolumn{2}{|c|}{ Husband's parenting cooperation } & .96 & - \\
\hline \multirow{4}{*}{ Support } & Emotional & \multirow{4}{*}{.97} & .93 \\
\hline & Physical & & .91 \\
\hline & Informative & & .90 \\
\hline & Appraisal & & .90 \\
\hline \multirow{3}{*}{ Parenting stress } & Parental distress & \multirow{3}{*}{.90} & .84 \\
\hline & Parent-child dysfunctional interaction & & .87 \\
\hline & Difficult child & & .75 \\
\hline
\end{tabular}

\subsection{Analysis method}

Using SPSS version 21.0, the collected data were analyzed through descriptive statistics, ttest, ANOVA, Pearson's correlation coefficient, and Hierological regression analysis.

\subsubsection{General characteristics}

The subjects were in their 30s, they graduated from university, the marriage period was less than three to five years, and the largest number of children raised was one. They were satisfied with their parenting environment, had no supportive caregiver, and were satisfied with their irregular workers and household income [Table 2].

Table 2. General characteristics $(N=279)$

\begin{tabular}{|c|c|c|}
\hline \multirow{4}{*}{ Age(year) } & Variable & $\mathrm{n}(\%)$ \\
\cline { 2 - 3 } & 20 's & $39(14.0 \%)$ \\
\cline { 2 - 3 } & 30 's & $223(79.9 \%)$ \\
\hline \multirow{4}{*}{ Education level } & 40 's & $17(6.1 \%)$ \\
\cline { 2 - 3 } & High school & $23(8.2 \%)$ \\
\cline { 2 - 3 } & College & $74(26.5 \%)$ \\
\cline { 2 - 3 } & University & $130(46.6 \%)$ \\
\hline \multirow{3}{*}{ Marriage period(year) } & $\geq$ Postgraduate school & $52(18.6 \%)$ \\
\cline { 2 - 3 } & $<1$ & $73(26.2 \%)$ \\
\cline { 2 - 3 } & $1 \sim<3$ & $83(29.7 \%)$ \\
\cline { 2 - 3 } & $3 \sim<5$ & $64(23.9 \%)$ \\
\hline \multirow{3}{*}{ Number of children } & $5 \leq$ & $59(21.1 \%)$ \\
\cline { 2 - 3 } & 1 & $158(56.6 \%)$ \\
\cline { 2 - 3 } & 2 & $101(36.2 \%)$ \\
\hline \multirow{2}{*}{ Environmental satisfaction } & Satisfaction & $20(7.2 \%)$ \\
\cline { 2 - 3 } & Unsatisfactory & $61(21.9 \%)$ \\
\hline Supportive caregiver & Y & $76(27.2 \%)$ \\
\hline
\end{tabular}




\begin{tabular}{|c|c|c|}
\hline & $\mathrm{N}$ & $203(72.8 \%)$ \\
\hline \multirow{2}{*}{ Retention facility } & Use & $172(61.6 \%)$ \\
\cline { 2 - 3 } & Not use & $107(38.4 \%)$ \\
\hline \multirow{2}{*}{ Job } & Full-time & $139(49.8 \%)$ \\
\cline { 2 - 3 } & Irregular workers & $140(50.2 \%)$ \\
\hline \multirow{3}{*}{ Income Satisfaction } & Very satisfied & $9(3.2 \%)$ \\
\cline { 2 - 3 } & Satisfaction & $149(53.4 \%)$ \\
\cline { 2 - 3 } & Unsatisfactory & $108(38.7 \%)$ \\
\cline { 2 - 3 } & Very dissatisfied & $13(4.7 \%)$ \\
\hline
\end{tabular}

\subsubsection{Differences in the degree of parenting stress according to the general characteristics of the subject}

The variables that showed significant differences in parents' distress were age and availability of retention facilities. According to the post-mortem analysis, parental distress was higher among parents in their 20s than in their 30s, and parental distress was higher than that among caregivers who did not use retention facilities [Table 3].

Table 3. Differences in parenting stress according to general characteristics

\begin{tabular}{|c|c|c|c|c|c|c|c|}
\hline \multirow{2}{*}{\multicolumn{2}{|c|}{ Variable }} & \multicolumn{2}{|c|}{ Parental distress } & \multicolumn{2}{|c|}{$\begin{array}{l}\text { Parent-child } \\
\text { dysfunctional } \\
\text { interaction }\end{array}$} & \multicolumn{2}{|c|}{ Difficult child } \\
\hline & & $M(S D)$ & $t / F$ & $M(S D)$ & $t / F$ & $M(S D)$ & $t / F$ \\
\hline \multirow{3}{*}{ Age(year) } & $20{ }^{\prime} s^{a}$ & $3.15(.57)$ & \multirow{3}{*}{$\begin{array}{c}5.230^{* * *} \\
(.006) \\
a>b\end{array}$} & $1.88(.55)$ & \multirow{3}{*}{$\begin{array}{l}1.507 \\
(.224)\end{array}$} & $2.42(.45)$ & \multirow{3}{*}{$\begin{array}{l}1.441 \\
(.238)\end{array}$} \\
\hline & 30 's ${ }^{b}$ & $2.84(.60)$ & & $1.80(.51)$ & & $2.35(.43)$ & \\
\hline & 40 's ${ }^{\mathrm{c}}$ & $2.69(.52)$ & & $1.62(.35)$ & & $2.21(.40)$ & \\
\hline \multirow{4}{*}{ Education level } & High school & $2.93(.65)$ & \multirow{4}{*}{$\begin{array}{l}0.439 \\
(.726)\end{array}$} & $1.82(.44)$ & \multirow{4}{*}{$\begin{array}{l}0.432 \\
(.730)\end{array}$} & $2.48(.35)$ & \multirow{4}{*}{$\begin{array}{l}1.858 \\
(.137)\end{array}$} \\
\hline & College & $2.86(.64)$ & & $1.77(.51)$ & & $2.39(.47)$ & \\
\hline & University & $2.84(.54)$ & & $1.79(.53)$ & & $2.29(.43)$ & \\
\hline & $\begin{array}{c}\geq \text { Postgraduate } \\
\text { school }\end{array}$ & 2.94(.67) & & $1.87(.49)$ & & $2.39(.42)$ & \\
\hline \multirow{4}{*}{$\begin{array}{c}\text { Marriage } \\
\text { period(year) }\end{array}$} & $<1$ & $22.97(.55)$ & \multirow{4}{*}{$\begin{array}{l}2.183 \\
(.090)\end{array}$} & $1.87(.45)$ & \multirow{4}{*}{$\begin{array}{l}1.698 \\
(.168)\end{array}$} & $2.37(.45)$ & \multirow{4}{*}{$\begin{array}{l}0.206 \\
(.892)\end{array}$} \\
\hline & $1 \sim<3$ & $2.92(.70)$ & & $1.83(.52)$ & & $2.36(.41)$ & \\
\hline & $3 \sim<5$ & $2.74(.61)$ & & $1.79(.58)$ & & $2.32(.45)$ & \\
\hline & $5 \leq$ & $2.81(.47)$ & & $1.68(.50)$ & & $2.36(.43)$ & \\
\hline \multirow{3}{*}{$\begin{array}{c}\text { Number of children } \\
\text { (person) }\end{array}$} & 1 & $2.89(.62)$ & \multirow{3}{*}{$\begin{array}{l}0.127 \\
(.881)\end{array}$} & $1.82(.47)$ & \multirow{3}{*}{$\begin{array}{l}0.403 \\
(.668)\end{array}$} & $2.34(.45)$ & \multirow{3}{*}{$\begin{array}{l}0.076 \\
(.927)\end{array}$} \\
\hline & 2 & $2.86(.58)$ & & $1.76(.60)$ & & $2.37(.41)$ & \\
\hline & $3 \leq$ & $2.83(.54)$ & & $1.79(.37)$ & & $2.35(.41)$ & \\
\hline \multirow{2}{*}{$\begin{array}{c}\text { Environmental } \\
\text { satisfaction }\end{array}$} & Satisfaction & $2.90(.59)$ & \multirow{2}{*}{$\begin{array}{l}-1.665 \\
(.097) \\
\end{array}$} & $1.81(.50)$ & \multirow{2}{*}{$\begin{array}{l}-0.620 \\
(.536) \\
\end{array}$} & $2.36(.45)$ & \multirow{2}{*}{$\begin{array}{l}-0.165 \\
(.869)\end{array}$} \\
\hline & Unsatisfactory & $2.75(.63)$ & & $1.76(.53)$ & & $2.35(.40)$ & \\
\hline \multirow{2}{*}{ Supportive caregiver } & $\mathrm{Y}$ & $2.92(.61)$ & \multirow{2}{*}{$\begin{array}{l}-0.856 \\
(.930)\end{array}$} & $2.36(.45)$ & \multirow{2}{*}{$\begin{array}{l}-0.755 \\
(.906) \\
\end{array}$} & $1.84(.52)$ & \multirow{2}{*}{$\begin{array}{r}-0.118 \\
(.451)\end{array}$} \\
\hline & $\mathrm{N}$ & $2.85(.60)$ & & $2.35(.43)$ & & $1.79(.51)$ & \\
\hline \multirow{2}{*}{ Retention facility } & Use & $2.80(.59)$ & \multirow{2}{*}{$\begin{array}{l}2.482^{*} \\
(.014)\end{array}$} & $2.36(.42)$ & \multirow{2}{*}{$\begin{array}{l}0.600 \\
(.679)\end{array}$} & $1.79(.54)$ & -0.414 \\
\hline & Not use & $2.98(.60)$ & & $2.34(.46)$ & & $1.82(.46)$ & $(.549)$ \\
\hline Joh & \begin{tabular}{|c|} 
Full-time \\
\end{tabular} & $2.89(.64)$ & -0.509 & $2.36(.46)$ & 0.023 & $1.80(.54)$ & -0.415 \\
\hline Job & Irregular workers & $2.85(.56)$ & $(.661)$ & $2.34(.40)$ & $(.981)$ & $1.80(.49)$ & $(.678)$ \\
\hline & \begin{tabular}{|l|} 
Very satisfied \\
\end{tabular} & $2.89(.49)$ & & $1.81(.29)$ & & $2.11(.32)$ & \\
\hline In & Satisfaction & $2.86(.61)$ & 0.242 & $1.79(.49)$ & 0.790 & $2.33(.43)$ & 1.868 \\
\hline Income Satisfaction & Unsatisfactory & $2.87(.59)$ & $(.889)$ & $1.79(.55)$ & $(.501)$ & $2.38(.44)$ & $(.135)$ \\
\hline & Vary dissatisfied & $3.01(.69)$ & & $2.01(.51)$ & & $2.52(.44)$ & \\
\hline
\end{tabular}




\subsubsection{Correlation between the parenting stress, parenting effectiveness, husband's parenting cooperation, and the degree of support}

A correlation analysis was conducted to identify the relationship between each variable. Increasing parenting stress has lowered parenting effectiveness, husband's parenting cooperation, and social support. In addition, if the effectiveness of parenting increased, the husband's parenting cooperation and social support increased. Support has increased as the husband's parenting cooperation increased [Table 4].

Table 3. Correlation and descriptive statistics of variables

\begin{tabular}{|c|c|c|c|c|c|c|c|c|c|c|c|c|c|c|c|}
\hline \multicolumn{2}{|c|}{ Variable } & \multicolumn{2}{|c|}{$\begin{array}{c}\mathrm{M} \\
(\mathrm{SD})\end{array}$} & 1 & 2 & 3 & 4 & 5 & 6 & 7 & 8 & 9 & 10 & 11 & 12 \\
\hline \multirow{3}{*}{$\begin{array}{c}\text { Parenting } \\
\text { stress }\end{array}$} & $\begin{array}{c}\text { Parenting } \\
\text { distress }^{1}\end{array}$ & \multirow{3}{*}{$\begin{array}{l}2.34 \\
(.41)\end{array}$} & $\begin{array}{l}2.87 \\
(.60)\end{array}$ & 1 & & & & & & & & & & & \\
\hline & $\begin{array}{l}\text { Parent-child } \\
\text { dysfunctiona } \\
\text { linteraction }^{2}\end{array}$ & & $\begin{array}{l}1.80 \\
(.51) \\
\end{array}$ & $.439^{* * * *}$ & & & & & & & & & & & \\
\hline & $\begin{array}{l}\text { Difficult } \\
\text { child }^{3}\end{array}$ & & $\begin{array}{l}2.35 \\
(.43)\end{array}$ & $.413^{* * *}$ & $.564^{* * *}$ & & & & & & & & & & \\
\hline \multirow{5}{*}{$\begin{array}{c}\text { Parenting } \\
\text { effective } \\
\text { ness }\end{array}$} & Affection ${ }^{4}$ & \multirow{5}{*}{$\begin{array}{l}4.38 \\
(.69)\end{array}$} & $\begin{array}{l}4.57 \\
(.72)\end{array}$ & $-.315^{* *}$ & $-.568^{* *}$ & $-.379^{* *}$ & & & & & & & & & \\
\hline & $\begin{array}{l}\text { Daily system } \\
\text { organization }\end{array}$ & & $\begin{array}{l}4.42 \\
(.85)\end{array}$ & $-.222^{* *}$ & $-.256^{* *}$ & $-.321^{* *}$ & $.420^{* * * *}$ & & & & & & & & \\
\hline & $\begin{array}{c}\text { Discipline } \\
6\end{array}$ & & $\begin{array}{l}4.29 \\
(.92)\end{array}$ & $-.434^{* *}$ & $-.595^{* *}$ & $-.553^{* *}$ & $.594^{* * *}$ & $.404^{* * *}$ & & & & & & & \\
\hline & Game $^{7}$ & & $\begin{array}{l}4.21 \\
(.89)\end{array}$ & $\begin{array}{c}-.344^{* *} \\
*\end{array}$ & $-.477^{* *}$ & $-.371^{* *}$ & $.630^{* * *}$ & $.407^{* * *}$ & $.528^{* * *}$ & & & & & & \\
\hline & Education $^{8}$ & & $\begin{array}{l}4.42 \\
(.94)\end{array}$ & $-.372^{* *}$ & $-.489^{* * *}$ & $-.492^{* *}$ & $.578^{* * * *}$ & $.653^{* * *}$ & $.643^{* * *}$ & $.515^{* * *}$ & & & & & \\
\hline \multicolumn{2}{|c|}{$\begin{array}{l}\text { Husband's } \\
\text { parenting } \\
\text { cooperation' }\end{array}$} & \multicolumn{2}{|c|}{$\begin{array}{l}3.69 \\
(.73)\end{array}$} & $\begin{array}{c}-.439^{* *} \\
*\end{array}$ & $-.355^{* * *}$ & $-.296^{* *}$ & $.464^{* * * *}$ & $.244^{* * *}$ & $.300^{* * *}$ & $.313^{* * *}$ & $.282^{* * * *}$ & & & & \\
\hline \multirow{4}{*}{ Support } & \begin{tabular}{|c|} 
Emotional \\
10 \\
\end{tabular} & \multirow{4}{*}{$\begin{array}{l}3.92 \\
(.66)\end{array}$} & $\begin{array}{l}3.87 \\
(.73) \\
\end{array}$ & $\begin{array}{c}-.217^{* *} \\
*\end{array}$ & $-\begin{array}{c}-.367^{* *} \\
*\end{array}$ & $-.189^{* *}$ & $.407^{* * * *}$ & $.245^{* * *}$ & $.238^{* * * *}$ & $.213^{* * *}$ & $.281^{* * *}$ & $.534^{* * *}$ & & & \\
\hline & Physical $^{11}$ & & $\begin{array}{l}3.96 \\
(.69)\end{array}$ & $\begin{array}{c}-.297^{* *} \\
*\end{array}$ & $\mid \begin{array}{c}-.392^{* *} \\
*\end{array}$ & $-.183^{* *}$ & $.394^{* * *}$ & $.247^{* * * *}$ & $.265^{* * *}$ & $.255^{* * *}$ & $.308^{* * *}$ & $.593^{* * * *}$ & $.887^{* * *}$ & & \\
\hline & $\begin{array}{c}\text { Information } \\
12\end{array}$ & & $\begin{array}{l}3.90 \\
(.70)\end{array}$ & $\begin{array}{c}-.233^{* *} \\
*\end{array}$ & $-.402^{* *}$ & $-.213^{* *}$ & $.425^{* * *}$ & $.240^{* * *}$ & $.241^{* * *}$ & $.244^{* * *}$ & $.313^{* * *}$ & $.520^{* * * *}$ & $.875^{* * *}$ & $.892^{* * *}$ & \\
\hline & $\underset{3}{\text { Appraisal }^{1}}$ & & $\begin{array}{l}3.93 \\
(.66)\end{array}$ & $-.341^{* *}$ & $-.448^{* *}$ & $-.250^{* *}$ & $.477^{* * * *}$ & $.270^{* * *}$ & $.328^{* * *}$ & $.302^{* * *}$ & $.334^{* * *}$ & $.620^{* * * *}$ & $.833^{* * *}$ & $894^{* * *}$ & $.831^{* * *}$ \\
\hline
\end{tabular}

2.4.4. The general characteristics of the subject, the effectiveness of the parenting, the husband's parenting cooperation, and the effect of support on parenting stress

For all models, the fit of the model is statistically significant $(\mathrm{p}<.001)$. During parenting stress, discipline effectiveness was a significant predictor of parental distress and husband's parenting cooperation. Parenting effectiveness and discipline were significant predictors of parent-child dysfunctional interactions. Difficult children were significant predictors of material support and discipline effectiveness [Table 5]. 
Table 5. Factors influencing parenting stress

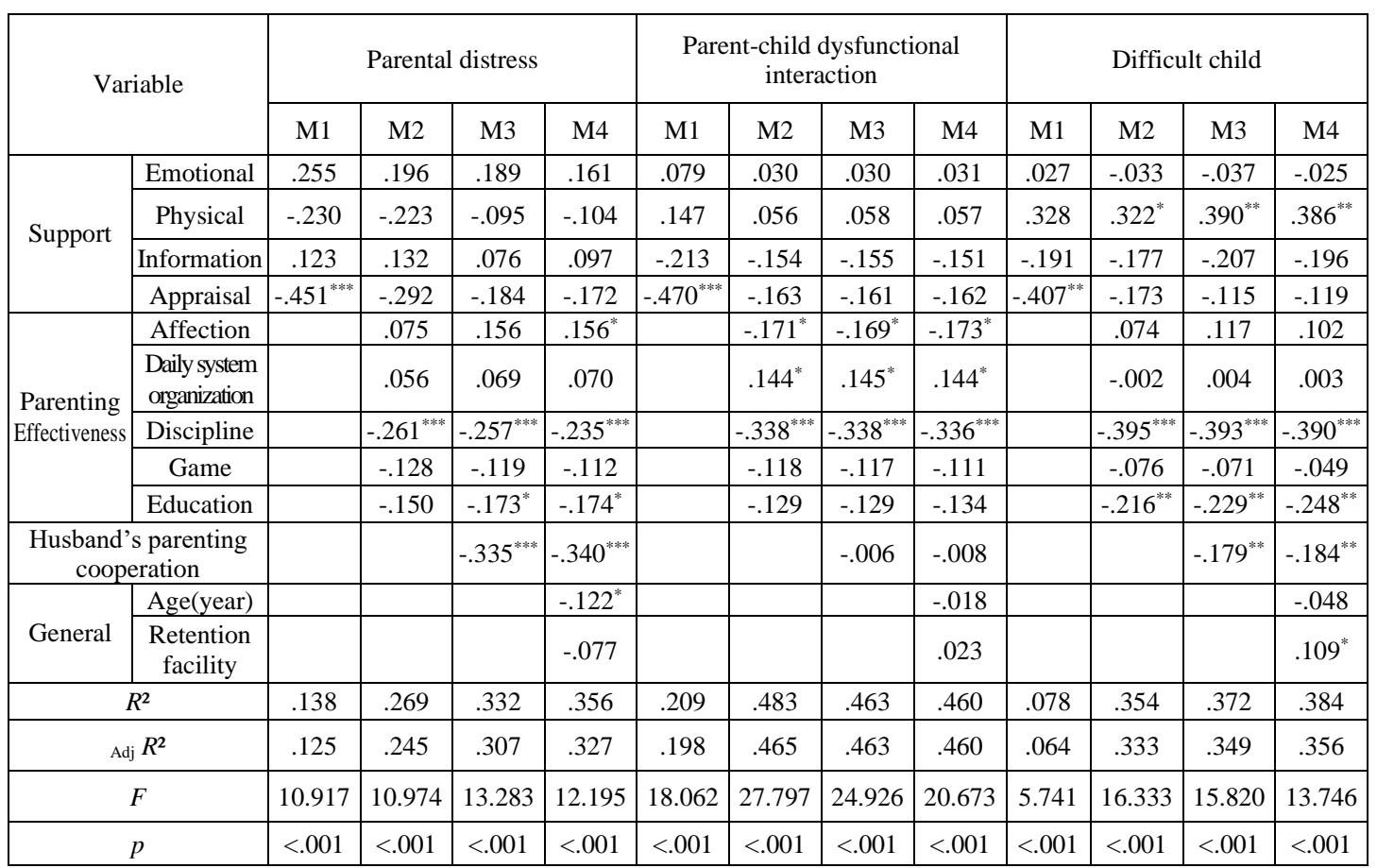

\section{Conclusions}

To achieve the objectives of this study, previous studies were reviewed to verify the influence of general characteristics, parenting effectiveness, husband's parenting cooperation, and support on a mother's parenting stress. To this end, data were collected and analyzed from an online community on parenting. Among the general characteristics, there were differences in parenting stress according to age and whether or not retention facilities were used. There was a significant positive correlation between parenting effectiveness, husband's parenting cooperation, and support. Material support, husband's parenting cooperation, affection effectiveness, and disciplinary efficacy were significant influencing factors on the mother's parenting stress.

Among the factors of parenting stress for mothers with infants and children, parental distress was influenced by the husband's parenting cooperation, and parent-child dysfunctional interaction and difficult children were influenced by parenting stress.

Specifically, parent distress has been identified as a significant influence on the husband's parenting cooperation and affection effectiveness. In the parent-child dysfunctional interaction, influencing factors for difficult children were identified as disciplinary effectiveness and material support. Therefore, environmental and resource provisions should be made such as expanding the number of childcare participants, educating parents about proper discipline, setting up parenting goals, identifying children's temperament, and sharing parenting information.

This study applied ecological theory [6] to confirm the factors influencing parenting stress. In a micro and intermediate system, a stable parenting environment should be provided centering on the family and mother, and that the child-rearing process is a common role for couples. And macrosystems will also be needed such as material support, mother's age, and 
retention facility use. Further research is needed as this study did not take into account the structure of single parents and other new forms of families.

\section{Reference}

[1] Statistics Korea, “2019 Women's life in statistics,” Daejeon, DJ: Springer pub Co., (2019)

[2] K. I. Park and S. Oh, "Effects of active parenting today based on goal attainment theory on parenting stress, parenting behavior, and parenting satisfaction in mothers of school-age children," Journal of Korean Academy of Nursing, vol.42, no.5, pp.659-670, (2012), DOI: 10.4040/jkan.2012.42.5.659

[3] K. M. Hasel, M. A. Besharat, A. Abdolhoseini, S. A. Nasab, and S. Niknam., "Relationships of personality factors to perceived stress, depression, and oral lichen planus severity," International Journal of Behavioral Medicine, vol.20, pp.286-292, (2013) DOI: 10.1007/s12529-012-9226-5

[4] Statistics Korea, “Korean social trends 2014,” Daejeon, DJ: Springer Pub Co., (2014)

[5] Y. H. Choi, and H. J. Moon., "The effects of parenting stress, parenting efficacy, spouse support and social support on parenting behavior of mothers with infant children," Early Childhood Education Research and Review, vol.20, no.6, pp.407-424, (2016)

[6] K. H. Kim and B. H. Cho, "An ecological approach to the analysis of variables in the parenting stress of the dual-earner mothers and fathers," The Korean Journal of Developmental Psychology, vol.24, no.24, pp.135150, (2011)

[7] J. H. Sung and J. H. Baek, "Validation of the Echelle globale du Sentiment de competence parentale (EGSCP) in Korea: A measure of domain-specific self-efficacy and related cognitive constructs for a parent," The Korean Journal of Developmental Psychology, vol.24, no.4, pp.135-150, (2011)

[8] E. Y. Choi, J. Choi, and S. H. Ahn, "The moderating effects of husbands' cooperation on the relationship between parenting stress and warm and accepting parenting behavior of working mothers," Journal of Korean Home Management Association, vol.36, no.4, pp.73-86, (2018) DOI: 10.7466/JKHMA.2018.36.4.73

[9] N. Y. Kim and H. H. Jeong, "The effects of art therapist's ego-resiliency and social support on psychological burnout," Korean Journal of art therapy, vol.19, no.4, pp.879-899, (2012) DOI: 10.35594/kata.2012.19.4.007

[10] K. M. Chung, Y. J. Yang, S. Jung, K. S. Lee, and J. A. Park., "Standardization study for the Korean version of parenting stress index fourth edition short form (K-PSI-4-SF)," The Korean Journal of Health Psychology, vol.27, no.3, pp.689-707, (2008) 
Factors Influencing the Parenting Stress of Mothers

This page is empty by intention. 